\title{
Reinvestigation of the Noncatalyzed Coupling of Aryllithium with Haloarene: A Novel Aromatic Nucleophilic Substitution Pathway
}

\author{
Jean-Michel Becht, Arnaud Gissot, Alain Wagner,* and Charles Mioskowski*[a]
}

\begin{abstract}
Noncatalyzed coupling reactions of aryllithiums and haloarenes proceed not only through the well-known aryne route but also, in some cases, through a novel addition - elimination pathway. Indeed, ortho-chloro- and ortho-bromomethoxyarenes lead selectively to the corresponding ortho-biaryls through a chelation-driven aromatic nucleophilic substitution pathway. Contrary to common belief, such noncatalyzed coupling reactions often proceed with high regioselectivity and high yield. These results underline the potency of such simple reactions and open up a straightforward access to a wide range of biaryl structures; this also appears particularly useful for large-scale and biaryl building-block syntheses, as only cheap and readily available substrates are involved.
\end{abstract}

Keywords: aromatic substitution - arynes - biaryls - metalation - nucleophilic substitution

\section{Introduction}

Biaryls are found in important biologically active molecules ${ }^{[1]}$ such as the naturally occurring terprenin ${ }^{[1]]}$ or in synthetic anti-HIV derivatives ${ }^{[1 g-i]}$ and are used as core structures for molecular recognition devices, ${ }^{[2]}$ organic semiconductors, material for nonlinear optics, and metal ligands for catalysts. $^{[3]}$ Several powerful synthetic methods are available for their preparation, ${ }^{[4]}$ including longknown reactions such as Ullmann ${ }^{[5]}$ or Gomberg - Bachmann - Hey couplings, ${ }^{[6]}$ or more modern reactions such as Stille, ${ }^{[7]}$ Suzuki Pd-catalyzed, and Ni-catalyzed cross-coupling reactions ${ }^{[8]}$ Although these reactions make it possible to prepare complex, polyfunctionalized biaryl structures under mild conditions, more efficient and simpler approaches are still suitable. Based on this analysis, we reinvestigated the noncatalyzed reaction of aryllithiums with haloarenes. Surprisingly, this reaction has only been little studied and very sparsely used in organic synthesis. The mechanism generally proposed in the literature proceeds via the in-situ formation of a highly reactive aryne intermediate ${ }^{[9]}$ that reacts with the aryllithium, leading to the biaryl structure. The mixture of regioisomers formed in the process is due to the two electrophilic positions present on the aryne intermediate. ${ }^{[10,11]}$ In addition to the classical mechanism involving arynes, another mechanism operates through competing halogen displacement and halogen - metal exchange processes. The reaction of phenyllithium, for example, with a 4-halotoluene leads to the formation of a complex mixture of the two possible meta and para isomers along with homocoupling reaction products arising from metal - halogen exchange side reactions ${ }^{[11]}$ These complex results proved discouraging for further synthetic developments, and stimulated no additional investigations or research in the field despite publication of three interesting reports. In 1983 Meyers succeeded in controlling the regioselectivity of the reaction by using an aryne precursor bearing a phenyloxazolidine substituent, which acts as a strong ortho-directing and chelating group. ${ }^{[12]}$ More recently, Schulte and Laschat found that 3,3',4,4'-tetraalkoxybiphenyl was obtained regioselectively by

${ }^{[a]}$ Dr. A. Wagner, Dr. C. Mioskowski, J.-M. Becht, A. Gissot Laboratoire de Synthèse Bioorganique, UMR 7514 associée au CNRS, Faculté de Pharmacie, 74 Route du Rhin, 67400 Illkirch (France) Fax: (33) 3-90-24-43-06, E-mail: wagner@bioorga.u-strasbg.fr, mioskowski@bioorga.u-strasbg.fr 
treatment of 4-bromo-1,2- dimethoxybenzene with 0.5 equiv of $n$-butyllithium in THF at $-78^{\circ} \mathrm{C}^{[13]}$ These $^{-1}$ results prompted us to reinvestigate the potential of this interesting transformation. We found that, in many cases, the reaction proceeds with high and predictable regioselectivity, making the transformation of interest for synthetic purposes. The substitution pattern of the haloarenes directs the formation of the biaryl structures, which are always obtained in good to moderate yields.

\section{Results and Discussion}

To evaluate the scope and limitations of this cross-coupling reaction we investigated the reactivity of several haloarenes with different aryllithiums. In a first set of experiments we used 1,3dimethoxybenzene as the anion precursor and chlorobenzene as the aryne source. Addition of $n$ butyllithium to a mixture of 1,3-dimethoxybenzene and chlorobenzene in various solvents (hexane, diethyl ether, THF, THF/HMPA) at various temperatures did not afford the desired biaryl structure. In contrast, good results were obtained when chlorobenzene was added to a solution of 2-lithio-1,3dimethoxybenzene in THF or DME and the resulting mixture was heated overnight at $60^{\circ} \mathrm{C}$. Under these conditions, the desired biaryl was obtained in $95 \%$ yield (Table 1, entry 2 ). At room temperature only poor yields are obtained. Notably, when the reaction was carried out on a $1.0 \mathrm{~mol}$ scale under the optimized conditions, the biaryl structure was obtained in a quantitative yield.

Table 1. Reaction of 2-lithio-1,3-dimethoxybenzene with halobenzene.

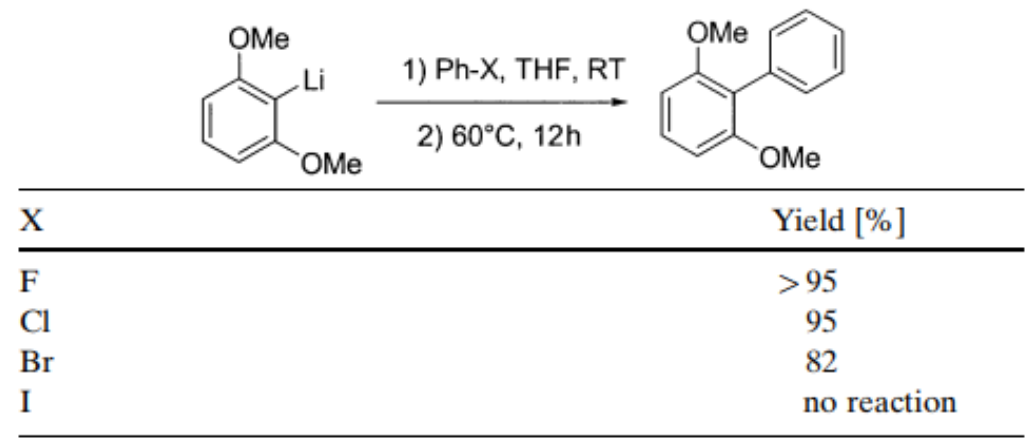

Next, we varied the nature of the halogen. The order of reactivity, $\mathrm{F}>\mathrm{Cl}>\mathrm{Br}>>\mathrm{I}$, is in accordance with a mechanism involving a transient aryne, the hydrogen abstraction being the rate-determining step. ${ }^{[11-14]}$ Interestingly, with iodobenzene no biaryl structure could be detected in the crude mixture by ${ }^{1} \mathrm{H}$ NMR analysis. The reaction was then extended to other aryllithiums generated under classical ortho-metalation conditions (Table 2).

Compared with 1,3-dimethoxybenzene (entry 2), the lower yields observed in the case of monostabilized anions (entries 1,3 ) might be due to the lower thermal stability of the 2-lithio anion. In accordance 1,2-dimethoxybenzene, which readily decomposes upon metalation, gave only traces of product (10\%). With phenyloxazoline, N,N-dimethylbenzamide and N-tert-butylbenzamide ortho anions, we observed a comparable lack of reactivity. To access a larger panel of biaryl structures, other aryllithiums were then generated by metal - halogen exchange (Table 3). ${ }^{[15]}$ 2-Lithioanisole was generated by bromine - lithium exchange from 2-bromoanisole by using metallic lithium (entry 1). ${ }^{[16]}$ Reaction with chlorobenzene led to the desired biaryl in 57\% yield. 4-Bromoanisole treated with tert-butyllithium gave the biaryl in 70\% yield. 4-Lithio-1,2- benzodioxole generated from 4-bromo-1,2-benzodioxole reacted with fluorobenzene to afford the coupling product in $42 \%$ yield (entry 3 ). 
Table 2. Reaction of various aryllithiums and chloro- or fluorobenzene

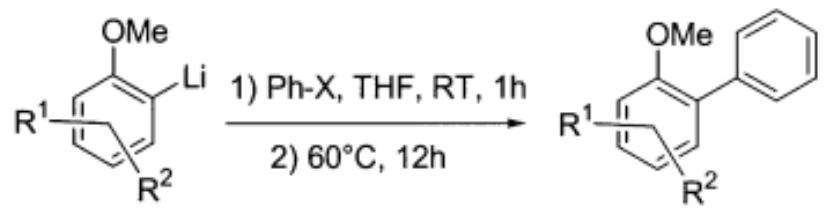

\begin{tabular}{lllll}
\hline Entry & $\mathrm{R}^{1}$ & $\mathrm{R}^{2}$ & $\mathrm{X}$ & Yield [\%] \\
\hline 1 & $\mathrm{H}$ & $\mathrm{H}$ & $\mathrm{F}$ & 48 \\
2 & $3-\mathrm{OMe}$ & $\mathrm{H}$ & $\mathrm{Cl}$ & 95 \\
3 & $4-\mathrm{OMe}$ & $\mathrm{H}$ & $\mathrm{F}$ & 52 \\
4 & $2-\mathrm{OMe}$ & $\mathrm{H}$ & $\mathrm{Cl}$ or F & - \\
5 & $3-\mathrm{OMe}$ & $5-\mathrm{OMe}$ & $\mathrm{F}$ & 55 \\
6 & $5-\mathrm{Me}$ & $\mathrm{H}$ & $\mathrm{F}$ & 41 \\
\hline
\end{tabular}

To extend the scope of this reaction, anions generated from $\alpha$-metalated heterocycles were tested (Scheme 1).
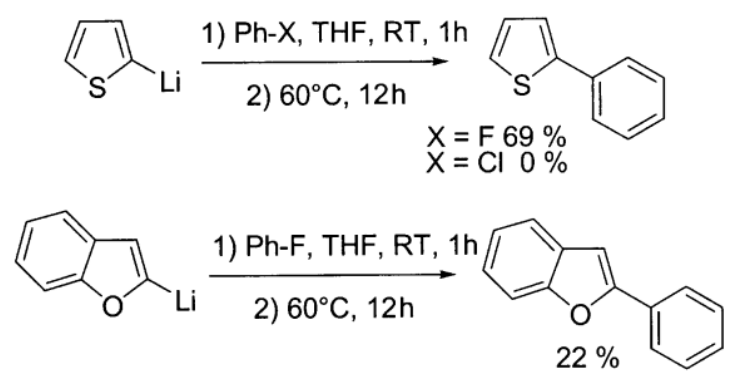

Scheme 1. Reaction of $\alpha$-metalated heterocycles and chloro- or fluorobenzene.

In both cases, only fluorobenzene allowed the reaction to proceed; coupling products were isolated in moderate $69 \%$ and low $22 \%$ yield. In this preliminary study, since only chloro- or fluorobenzene was used as the aryne precursor, the regioselectivity issue was avoided. To address this issue, we employed functionalized haloarenes for which random aryne formation and/or subsequent random nucleophilic attack are expected to lead to a mixture of isomers (Scheme 2). 


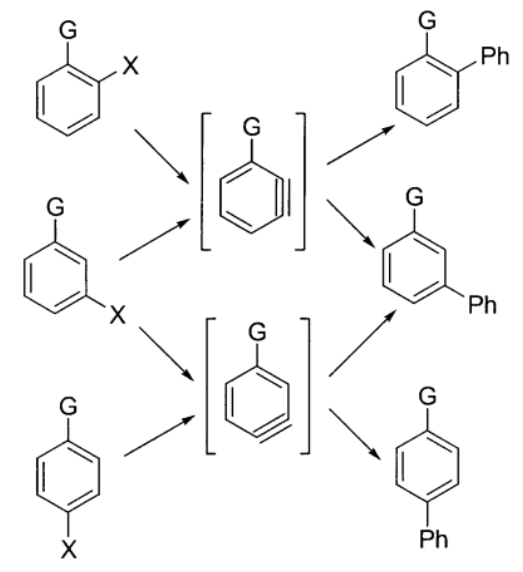

Scheme 2. Possible products formed through random aryne formation and subsequent random nucleophilic attack.

Table 4. Reaction of 2-lithio-1,3-dimethoxybenzene with substituted chloro- and fluorobenzene.

\begin{tabular}{clllllll} 
& & & & & & \\
\hline
\end{tabular}

Reaction of 2-, 3-, and 4-haloanisoles with 2-lithio-1,3- dimethoxybenzene (Table 4) was studied first. The ortho/ meta/para-biaryl ratios were determined by GC analysis of the crude mixtures. The ortho and para isomers were identified unambiguously by comparison with reference samples synthesized by classical Pd coupling reactions. ${ }^{[8]}$ The structures of the meta isomers were assessed by X-ray analysis. Reaction of 2lithio-1,3-dimethoxybenzene with 2-chloroanisole gave the ortho-biaryl in $80 \%$ yield and $82 \%$ selectivity (entry 1 ), whereas 3 -chloroanisole led predominantly to the meta isomer with $97 \%$ selectivity and $82 \%$ overall yield (entry 4). Exclusive formation of a transient 2,3-aryne, followed by addition of the aryllithium specifically at the 3-position, leads to the most stabilized anionic intermediate. This set of steps accounts for the formation of the meta-biaryl obtained from 3-chloroanisole. According to this mechanism, 2- 
chloroanisole is expected to give the same 2,3-aryne intermediate and thus the same reaction product. Instead, the observed formation of the ortho-biaryl isomer, in total contradiction of the classical arynebased mechanism, suggests a novel aromatic nucleophilic substitution pathway (Scheme 3).

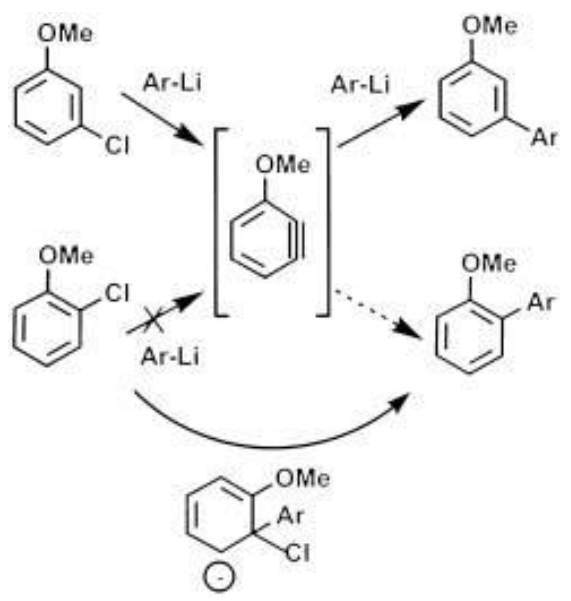

Scheme 3. Possible mechanism for the reaction of 2- and 3-chloroanisole.

To support this hypothesis, substitution of 2-chloroanisole by 2-fluoroanisole reversed the reaction selectivity (entry 2). The strong ortho-acidifying effect of the fluorine atom favors hydrogen abstraction and thus the aryne pathway. In accordance with this, 2-fluoroanisole yielded the meta isomer cleanly. In contrast, since the bromine atom has little or no acidifying effect, with 2-bromoanisole nucleophilic substitution took over and the ortho isomer was obtained in $75 \%$ yield (entry 3 ). 4-Chloroanisole afforded a 1:1 mixture of the two possible meta and para isomers, as a result of formation of the 3,4- aryne and subsequent random nucleophilic attack at positions 3 and 4 (entry 5). To check whether this regioselectivity was specific for the anion of 1,3-dimethoxybenzene, we carried out experiments under similar conditions using phenyllithium. 2-Chloroanisole afforded the ortho-biaryl in 78\% isolated yield and 3 -chloroanisole the meta isomer in $80 \%$ isolated yield. These results indicate clearly that the selectivity is mainly governed by the substitution pattern and the nature of the haloarenes. Other substrates, such as 2-chloro- and 3-chloro-N,Ndimethylanilines (entries 6, 7), afforded exclusively metabiphenyl in $40 \%$ and $47 \%$ yield respectively. Replacement of the chlorine atom by a less acidifying bromine atom (entry 8 ) did not change the selectivity, but resulted in a dramatic drop in the yield. Similar results were obtained with phenyllithium. Results observed with the halotoluenes (entries 9 - 14) are in total accordance with the aryne pathway and with an early report by Chlebowski. ${ }^{[11]}$ Here again, the increase in yield by switching from chloro- to fluorotoluene indicates that $\alpha$-hydrogen abstraction is a determining step in the mechanism (Table 1). The slight deviation from the statistical ratio of the possible regioisomers might be explained by the steric hindrance of the methyl group, which disfavors the nucleophilic addition at the 2position. 3-Chlorotrifluoromethylbenzene gave a mixture of meta and para isomers in a 7:3 ratio (entry 15), which differs from the ratio obtained with 3-chlorotoluene (entry 12). The better ortho-stabilizing effect of the trifluoromethyl group accounts for this result. 4-Chlorotrifluoromethylbenzene gave a statistical mixture of meta- and para-biaryls (entry 16). To prove the ipso nucleophilic substitution pathway, we synthesized 2-chloro-3-deuteroanisole. meta-Anisidine was first converted into 2-chloro-3aminoanisole, ${ }^{[17 \mathrm{a}]}$ which was diazotized ${ }^{[17 b]}$ and reduced with sodium borodeuteride ${ }^{[17 \mathrm{~d}]}$ to afford the desired deuterated substrate. The latter was employed in an anionic cross-coupling reaction (Scheme 4). 


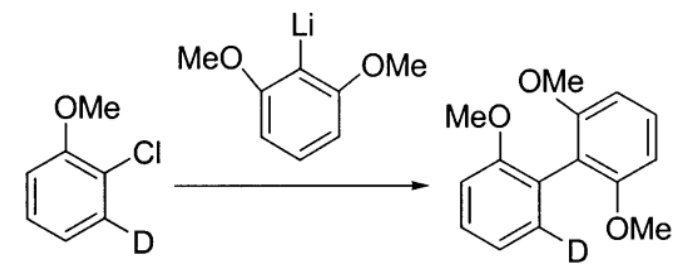

Scheme 4. Ipso substitution reaction on deuterated substrate.

As expected, 2-chloro-3-deuteroanisole leads to the coupling product with less than $5 \%$ of deuterium loss, showing that no hydrogen abstraction occurs. This result clearly demonstrates that, for this isomer, the biaryl structure is formed through an ipso nucleophilic substitution mechanism. Next, we tested the hypothesis that the selectivity could result from the chelation of the lithium cation by the oxygen atom of the methoxy group. Addition of HMPA, which is known to disrupt the lithium chelation, resulted in the reversal of selectivity with formation of the meta isomer in $68 \%$ yield. Similarly, when the reaction was carried out in the presence of $\mathrm{LiClO}_{4}$ both the ortho and the meta isomers were obtained in a 55:45 ratio. It thus appears that lithium chelation has a predominant role in directing the ipso substitution. Such chelation-driven selectivity also accounts for the experimental results obtained with 2-haloaniline (Table 4, entries $6-8$ ), for which the biaryl formation proceeds through the aryne pathway since the nitrogen atom has weak lithium chelation ability. Even though a chelation-driven addition of phenylmagnesium bromide to ortho-methoxyphenyloxazoline was described recently, ${ }^{[18]}$ to the best of our knowledge this unusual mechanistic pathway has been neither studied nor exploited in organic synthesis.

To extend its scope further, we considered haloarenes bearing two directing groups (Table 5).

Table 5. Reaction of 2-lithio-1,3-dimethoxybenzene with di-substituted chloro- and fluorobenzene derivatives.

\begin{tabular}{lllllll} 
& & & \\
\hline
\end{tabular}

These results indicate that the rules governing the reaction pathways are perfectly understood and predictable. The nucleophilic substitution is preferred when possible; if it is not possible, the major isomer arises from the most stable anion adduct (Scheme 5). 

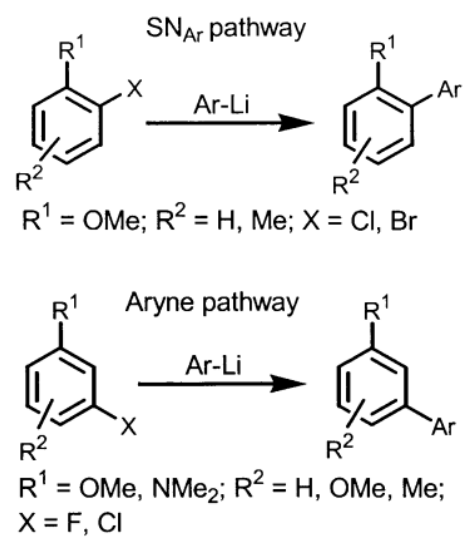

Scheme 5. Summary of the substrate substitution reaction pathways.

In conclusion, we have shown that noncatalyzed coupling of aryllithiums with haloarene often proceeds with high selectivity. ortho-Chloro- and ortho-bromomethoxyarenes give the corresponding ortho-biaryls through chelation-driven aromatic nucleophilic substitution. For other substrates the biaryl comes from the addition of the aryllithium to an aryne intermediate at the position leading to the most stable anionic adduct. For haloarenes bearing substituents which do not stabilize the anionic adduct, a statistical mixture of the possible isomers is obtained. For substrates bearing two stabilizing groups, the regioselectivity is governed by the strongest directing group. These noncatalyzed reactions give a straightforward access to a wide range of useful biaryl structures. As only cheap and readily available substrates are involved, our process appears to be of special interest for large-scale synthesis of basic biaryl building blocks. To pioneer a novel type of aromatic chemistry, such chelation-driven substitution might be extendable to other carbanionic nucleophiles. Studies are currently being undertaken in that direction.

\section{Experimental Section}

General: ${ }^{1} \mathrm{H}$ and ${ }^{13} \mathrm{C} \mathrm{NMR}$ spectra in $\mathrm{CDCl} 3$ were recorded using either a $200 \mathrm{MHz}$ or a $300 \mathrm{MHz}$ instrument. Chemical $(\delta)$ shifts are reported in parts per million downfield from TMS. Gas chromatography was performed on a Carlo Erba 6000 Vega equipped with a flame ionization detector using a $25 \mathrm{~m} \mathrm{BPX} 5$ fused silica column. The GC conditions for isomer analysis were: pressure of carrier $20 \mathrm{kPa}$; injection of the sample at $170^{\circ} \mathrm{C}$ kept constant for $3 \mathrm{~min}$ then increased to $240^{\circ} \mathrm{C}$ at $40^{\circ} \mathrm{C} \cdot \mathrm{min}^{-1}$ for $17 \mathrm{~min}$. Tetrahydrofuran (THF) was distilled from sodium/benzophenone. Dimethoxyethane (DME) was dried with molecular sieves. All biaryl syntheses by our methodology were performed in flame-dry glassware under an argon atmosphere. Since the descriptions of the various biaryl structures are dispersed and incomplete in the literature, we give full characterizations of all the synthesized compounds.

General procedure for synthesis of the biaryls in Tables 1, 4, and 5: n-Butyllithium (1.6M solution in hexane, $3.9 \mathrm{~mL}, 6.3 \mathrm{mmol}$ ) was added dropwise at RT to a solution of 1,3-dimethoxybenzene (6.3 mmol) in THF $(5 \mathrm{~mL})$. After $1 \mathrm{~h}$ a solution of aryl halide $(2.9 \mathrm{mmol})$ in THF $(2 \mathrm{~mL})$ was added dropwise. The resulting mixture was stirred for another hour at RT and then heated at $60^{\circ} \mathrm{C}$ for $12 \mathrm{~h}$. The reaction mixture was cooled to RT and quenched by addition of $\mathrm{H}_{2} \mathrm{O}(40 \mathrm{~mL})$. The aqueous layer was extracted twice with $\mathrm{Et}_{2} \mathrm{O}$ 
(total $60 \mathrm{~mL}$ ), and the combined organic layers were dried over $\mathrm{MgSO}_{4}$, filtered, and concentrated under vacuum. The residue was purified by silica gel flash chromatography with $\mathrm{Et}_{2} \mathrm{O} / \mathrm{hexane}$. If they were still colored after chromatographic purification, the products were filtered on charcoal.

2,6-Dimethoxybiphenyl (Table 1 and Table 2, entry 2): White solid, m.p. $89^{\circ} \mathrm{C} ; R_{f} 0.5\left(10 \% \mathrm{Et}_{2} \mathrm{O} /\right.$ hexane); ${ }^{1} \mathrm{H} \mathrm{NMR}\left(200 \mathrm{MHz},\left[\mathrm{D}_{1}\right] \mathrm{CHCl}_{3}, 20^{\circ} \mathrm{C}\right): 3.83\left(\mathrm{~s}, 6 \mathrm{H} ; 2 \mathrm{CH}_{3}\right), 6.76\left(\mathrm{~d},{ }^{3} \mathrm{~J}(\mathrm{H}, \mathrm{H}) 8.8 \mathrm{~Hz}, 2 \mathrm{H} ; 3,5-\mathrm{H}\right), 7.39\left(\mathrm{t},{ }^{3} \mathrm{~J}(\mathrm{H}, \mathrm{H})\right.$ $8.8 \mathrm{~Hz}, 1 \mathrm{H} ; 4-\mathrm{H}), 7.49-7.59 \mathrm{ppm}(\mathrm{m}, 5 \mathrm{H} ; 5-\mathrm{H}) ;{ }^{13} \mathrm{C} \mathrm{NMR}\left(50 \mathrm{MHz},\left[\mathrm{D}_{1}\right] \mathrm{CHCl}_{3}, 20^{\circ} \mathrm{C}\right): 55.8,104.1,119.4$, 126.7, 127.6, 128.5, 130.8, 134.1, 157.6 ppm; IR $\left(\mathrm{CHCl}_{3}\right): \bar{v}=3054,2946,1584,1470,1427,1242,1103 \mathrm{~cm}^{-}$ 1; $\mathrm{MS}\left(\mathrm{NH}_{4}^{+}\right): \mathrm{m} / \mathrm{z}: 232\left[\mathrm{M}+\mathrm{NH}_{4}{ }^{+}\right]$; elemental analysis calcd (\%) for $\mathrm{C}_{14} \mathrm{H}_{14} \mathrm{O}_{2}$ (214.26): C 78.48, $\mathrm{H} \mathrm{6.58,} \mathrm{O}$ 14.94; found: C 78.32, H 6.56, O 14.89.

2-Methoxybiphenyl (Table 2, entry 1): After a solution of 2-bromoanisole (15 mmol) in $\mathrm{Et}_{2} \mathrm{O}(16.8 \mathrm{~mL}) \mathrm{had}$ been added dropwise at RT to a suspension of lithium wires ( $400 \mathrm{mg}, 58 \mathrm{mmol}$ ) in $\mathrm{Et}_{2} \mathrm{O}(16.8 \mathrm{~mL}$ ), the medium was stirred for $2 \mathrm{~h}$ at RT, then a portion $(16.8 \mathrm{~mL})$ of this solution was diluted with THF $(8 \mathrm{~mL})$. A solution of fluorobenzene $(2.4 \mathrm{mmol})$ in THF $(2 \mathrm{~mL})$ was added dropwise at RT. The reaction mixture was stirred for $1 \mathrm{~h}$ at RT and refluxed to $60^{\circ} \mathrm{C}$ for $12 \mathrm{~h}$. The reaction mixture was allowed to cool to RT and quenched by addition of water $\left(40 \mathrm{~mL}\right.$ ). The aqueous layer was extracted twice with $\mathrm{Et}_{2} \mathrm{O}$ (total $100 \mathrm{~mL}$ ), then the combined organic layers were dried over $\mathrm{MgSO}_{4}$, filtered, concentrated in vacuo, and purified by flash chromatography on silica gel with hexane as eluent. Yield $250 \mathrm{mg}(57 \%)$.

2-Methoxybiphenyl (Table 2, entry 1): Colorless oil; $R_{f} 0.5$ (10\% AcOEt/hexane); ${ }^{1} \mathrm{H}$ NMR (200 MHz, [ $\left.\mathrm{D}_{1}\right]$ $\left.\mathrm{CHCl}_{3}, 20^{\circ} \mathrm{C}\right): 3.98\left(\mathrm{~s}, 3 \mathrm{H} ; \mathrm{OCH}_{3}\right), 7.15-7.26(\mathrm{~m}, 2 \mathrm{H} ; 3,5-\mathrm{H}), 7.52-7.79 \mathrm{ppm}(\mathrm{m}, 7 \mathrm{H} ; 4,6-\mathrm{H}, 5 \mathrm{H}) ;{ }^{13} \mathrm{C}$ NMR (50 MHz, [ $\left.\mathrm{D}_{1}\right] \mathrm{CHCl}_{3}, 20^{\circ} \mathrm{C}$ ): 56.2, 112.0, 121.6, 127.7, 128.8, 129.4, 130.3, 131.4, 131.6, 139.4, $157.2 \mathrm{ppm}$; IR $\left(\mathrm{CHCl}_{3}\right): \bar{v}=3060,2926,1599,1499,1482,1258,1025 \mathrm{~cm}^{-1} ; \mathrm{MS}\left(\mathrm{NH}_{4}^{+}\right): \mathrm{m} / \mathrm{z}: 202\left[\mathrm{M}+\mathrm{NH}_{4}^{+}\right]$; elemental analysis calcd (\%) for $\mathrm{C}_{13} \mathrm{H}_{12} \mathrm{O}$ (184.24): C 84.75, H 6.56, O 8.68; found: C 84.56, H 6.54, 0 8.71.

General procedure for the synthesis of biaryls (Table 3): The bromoaryl $\left(5.4 \mathrm{mmol}\right.$ ) was added at $78^{\circ} \mathrm{C}$ to a solution of t-BuLi (1.7 in pentane, $6.4 \mathrm{~mL}, 10.8 \mathrm{mmol}$ ). The reaction mixture was warmed to RT for 30 min. After it had been cooled to $0^{\circ} \mathrm{C}$, DME $(3 \mathrm{~mL})$ and THF $(3 \mathrm{~mL})$ were added successively. Halobenzene $(1.5 \mathrm{mmol})$ was diluted in DME $(2.5 \mathrm{~mL})$, and the resulting solution was heated at $70^{\circ} \mathrm{C}$; a solution of aryllithium $(10.4 \mathrm{~mL})$ was added quickly at $70^{\circ} \mathrm{C}$. The reaction mixture was stirred at this temperature for $12 \mathrm{~h}$. After cooling to RT, the reaction was quenched by addition of water $(40 \mathrm{~mL})$. The aqueous layer was extracted twice with $\mathrm{Et}_{2} \mathrm{O}$ (total $100 \mathrm{~mL}$ ). The combined organic layers were dried over $\mathrm{MgSO}_{4}$, filtered, and concentrated under vacuum. The residue was purified by flash chromatography on silica gel.

2,5-Dimethoxybiphenyl (Table 2, entry 3): Colorless oil; $\mathrm{R}_{\mathrm{f}} 0.4$ (10\% $\mathrm{Et}_{2} \mathrm{O} /$ hexane); ${ }^{1} \mathrm{H} \mathrm{NMR}(300 \mathrm{MHz}$, $\left.\left[\mathrm{D}_{1}\right] \mathrm{CHCl}_{3}, 20^{\circ} \mathrm{C}\right): 3.77\left(\mathrm{~s}, 3 \mathrm{H} ; \mathrm{OCH}_{3}\right), 3.83\left(\mathrm{~s}, 3 \mathrm{H} ; \mathrm{OCH}_{3}\right), 6.84-6.96(\mathrm{~m}, 3 \mathrm{H} ; 3,4,6-\mathrm{H}), 7.34$ - $7.61 \mathrm{ppm}(\mathrm{m}$, $5 \mathrm{H} ; 5 \mathrm{H}) ;{ }^{13} \mathrm{C} \mathrm{NMR}\left(75 \mathrm{MHz},\left[\mathrm{D}_{1}\right] \mathrm{CHCl}_{3}, 20^{\circ} \mathrm{C}\right): 55.8,56.3,112.7,113.1,116.7,127.1,128.0,129.4,131.7$, 138.4, 150.8, 153.8 ppm; IR $\left(\mathrm{CHCl}_{3}\right): \bar{v}=3069,2944,1601,1505,1488,1216,1040 \mathrm{~cm}^{-1} ; \mathrm{MS}\left(\mathrm{NH}_{4}^{+}\right): \mathrm{m} / \mathrm{z}:$ $232\left[\mathrm{M}+\mathrm{NH}_{4}^{+}\right]$; elemental analysis calcd (\%) for $\mathrm{C}_{14} \mathrm{H}_{14} \mathrm{O}_{2}$ (214.26): C 78.48, $\mathrm{H} 6.58, \mathrm{O}$ 14.93; found: C 78.39, H 6.60, O 14.89.

2,4,6-Trimethoxybiphenyl (Table 2, entry 5): White solid, m.p. $152^{\circ} \mathrm{C} ; \mathrm{R}_{\mathrm{f}} 0.4$ (20\% $\mathrm{Et}_{2} \mathrm{O} /$ hexane); ${ }^{1} \mathrm{H}$ NMR $\left(300 \mathrm{MHz},\left[\mathrm{D}_{1}\right] \mathrm{CHCl}_{3}, 20^{\circ} \mathrm{C}\right): 3.74\left(\mathrm{~s}, 6 \mathrm{H} ; 2 \mathrm{OCH}_{3}\right), 3.89\left(\mathrm{~s}, 3 \mathrm{H} ; \mathrm{OCH}_{3}\right), 6.26(\mathrm{~s}, 2 \mathrm{H} ; 3,5-\mathrm{H}), 7.28-7.44 \mathrm{ppm}$ $(\mathrm{m}, 5 \mathrm{H} ; 5 \mathrm{H}) ;{ }^{13} \mathrm{C} \mathrm{NMR}\left(75 \mathrm{MHz},\left[\mathrm{D}_{1}\right] \mathrm{CHCl}_{3}, 20^{\circ} \mathrm{C}\right): 55.3,55.9,90.9,126.4,127.6,130.9,131.2,134.1,158.3$, 160.5 ppm; IR $\left(\mathrm{CHCl}_{3}\right): \bar{v}=3020,2930,1607,1484,1461,1211,1127 \mathrm{~cm}^{-1} ; \mathrm{MS}\left(\mathrm{NH}_{4}^{+}\right): \mathrm{m} / \mathrm{z}: 245\left[\mathrm{M}+\mathrm{H}^{+}\right]$; 
elemental analysis calcd (\%) for $\mathrm{C}_{15} \mathrm{H}_{16} \mathrm{O}_{3}$ (244.29): C 73.75, H 6.60, O 19.65; found: C 73.98, H 6.57, O 19.70 .

2-Methoxy-5-methylbiphenyl (Table 2, entry 6): Colorless oil; $\mathrm{R}_{\mathrm{f}} 0.5$ (4\% $\mathrm{Et}_{2} \mathrm{O} /$ hexane); ${ }^{1} \mathrm{H}$ NMR (300 $\left.\mathrm{MHz},\left[\mathrm{D}_{1}\right] \mathrm{CHCl}_{3}, 20^{\circ} \mathrm{C}\right): 2.43\left(\mathrm{~s}, 3 \mathrm{H} ; \mathrm{CH}_{3}\right), 3.85\left(\mathrm{~s}, 3 \mathrm{H} ; \mathrm{OCH}_{3}\right), 6.96\left(\mathrm{~d},{ }^{3} \mathrm{~J}(\mathrm{H}, \mathrm{H}) 8.4 \mathrm{~Hz}, 1 \mathrm{H} ; 3-\mathrm{H}\right), 7.19-7.27$ $(\mathrm{m}, 2 \mathrm{H} ; 4,6-\mathrm{H}), 7.39-7.64 \mathrm{ppm}(\mathrm{m}, 5 \mathrm{H} ; 5 \mathrm{H}) ;{ }^{13} \mathrm{C} \mathrm{NMR}\left(75 \mathrm{MHz},\left[\mathrm{D}_{1}\right] \mathrm{CHCl}_{3}, 20^{\circ} \mathrm{C}\right): 20.4,55.7,111.3,126.8$, $127.9,128.8,129.5,129.9,130.5,131.6,138.6,154.4$ ppm; IR $\left(\mathrm{CHCl}_{3}\right): \bar{v}=3025,2936,1603,1508,1490$, 1236, $1029 \mathrm{~cm}^{-1}$; $\mathrm{MS}\left(\mathrm{NH}_{4}^{+}\right): \mathrm{m} / \mathrm{z}: 216\left[\mathrm{M}+\mathrm{NH}_{4}{ }^{+}\right]$; elemental analysis calcd (\%) for $\mathrm{C}_{14} \mathrm{H}_{14} \mathrm{O}$ (198.26): C 84.81, H 7.11, O 8.08; found: C 84.67, H 7.10, 0 8.05.

4-Methoxybiphenyl (Table 3, entry 2): White solid, m.p. 89 ${ }^{\circ} \mathrm{C} ; \mathrm{R}_{\mathrm{f}} 0.3$ (hexane); ${ }^{1} \mathrm{H}$ NMR (200 MHz, $\left[\mathrm{D}_{1}\right]$ $\left.\mathrm{CHCl}_{3}, 20^{\circ} \mathrm{C}\right): 3.90\left(\mathrm{~s}, 3 \mathrm{H} ; \mathrm{OCH}_{3}\right), 7.03\left(\mathrm{~d},{ }^{3} \mathrm{~J}(\mathrm{H}, \mathrm{H}) 8.8 \mathrm{~Hz}, 2 \mathrm{H} ; 3,5-\mathrm{H}\right), 7.31-7.66 \mathrm{ppm}(\mathrm{m}, 7 \mathrm{H} ; 2,6-\mathrm{H}, 5 \mathrm{H}) ;{ }^{13} \mathrm{C}$ NMR $\left(75 \mathrm{MHz},\left[\mathrm{D}_{1}\right] \mathrm{CHCl}_{3}, 20^{\circ} \mathrm{C}\right)$ : 55.2, 114.1, 126.6, 126.7, 128.1, 128.7, 133.7, 140.8, $159.1 \mathrm{ppm}$; IR $\left(\mathrm{CHCl}_{3}\right) \bar{v}=3050,2958,1606,1516,1485,1272,1037 \mathrm{~cm}^{-1} ; \mathrm{MS}\left(\mathrm{NH}_{4}^{+}\right): \mathrm{m} / \mathrm{z}: 185\left[\mathrm{M}+\mathrm{H}^{+}\right]$; elemental analysis calcd (\%) for $\mathrm{C}_{13} \mathrm{H}_{12} \mathrm{O}$ (184.24): C 84.75, H 6.56, O 8.68; found: C 84.99, H 6.54, O 8.65.

2,6,2-Trimethoxybiphenyl (Table 4, entries 1, 3): White solid, m.p. $141^{\circ} \mathrm{C} ; \mathrm{R}_{\mathrm{f}} 0.2$ (10\% AcOEt/hexane); GC retention time $9.29 \mathrm{~min} ;{ }^{1} \mathrm{H} \mathrm{NMR}\left(200 \mathrm{MHz},\left[\mathrm{D}_{3}\right] \mathrm{CH}_{3} \mathrm{CN}, 20^{\circ} \mathrm{C}\right): 3.67\left(\mathrm{~s}, 6 \mathrm{H} ; 2 \mathrm{OCH}_{3}\right), 3.70\left(\mathrm{~s}, 3 \mathrm{H} ; \mathrm{OCH}_{3}\right)$, $6.70\left(\mathrm{~d},{ }^{3} \mathrm{~J}(\mathrm{H}, \mathrm{H}) 8.3 \mathrm{~Hz}, 2 \mathrm{H} ; 3,5-\mathrm{H}\right), 6.90$ - $7.10(\mathrm{~m}, 3 \mathrm{H} ; 3,5,6-\mathrm{H}), 7.25$ - 7.35 ppm (m, 2H; 4,4-H); ${ }^{13} \mathrm{C}$ NMR (75 MHz, $\left[\mathrm{D}_{3}\right] \mathrm{CH}_{3} \mathrm{CN}, 20^{\circ} \mathrm{C}$ ): 56.1, 56.4, 105.2, 112.1, 121.1, 124.9, 129.4, 129.9, 133.0, 158.6, 159.0 ppm; IR $\left(\mathrm{CHCl}_{3}\right): \bar{v}=3008,2938,1591,1504,1471,1250,1111 \mathrm{~cm}^{-1} ; \mathrm{MS}\left(\mathrm{NH}_{4}^{+}\right): \mathrm{m} / \mathrm{z}: 245\left[\mathrm{M}+\mathrm{H}^{+}\right]$; elemental analysis calcd (\%) for $\mathrm{C}_{15} \mathrm{H}_{16} \mathrm{O}_{3}$ (244.29): C 73.75, H 6.60, O 19.65; found: C 73.71, H 6.61, O 19.61.

2,6,3'-Trimethoxybiphenyl (Table 4, entries 2, 4): White solid, m.p. $70^{\circ} \mathrm{C} ; \mathrm{R}_{\mathrm{f}} 0.2$ (5\% $\mathrm{Et}_{2} \mathrm{O} /$ hexane); GC retention time $11.16 \mathrm{~min} ;{ }^{1} \mathrm{H} \mathrm{NMR}\left(200 \mathrm{MHz},\left[\mathrm{D}_{1}\right] \mathrm{CHCl}_{3}, 20^{\circ} \mathrm{C}\right): 3.75\left(\mathrm{~s}, 6 \mathrm{H} ; 2 \mathrm{OCH}_{3}\right), 3.83\left(\mathrm{~s}, 3 \mathrm{H} ; \mathrm{OCH}_{3}\right)$, $6.66\left(\mathrm{~d},{ }^{3} \mathrm{~J}(\mathrm{H}, \mathrm{H}) 7.8 \mathrm{~Hz}, 2 \mathrm{H} ; 3,5-\mathrm{H}\right), 6.85-6.97(\mathrm{~m}, 3 \mathrm{H} ; 2,4,6-\mathrm{H}), 7.25$ - $7.38 \mathrm{ppm}(\mathrm{m}, 2 \mathrm{H} ; 4,5-\mathrm{H}) ;{ }^{13} \mathrm{C}$ NMR (75 MHz, $\left[\mathrm{D}_{1}\right] \mathrm{CHCl}_{3}, 20^{\circ} \mathrm{C}$ ): 55.1, 55.9, 104.2, 112.4, 113.2, 116.6, 123.3, 128.5, 131.9, 135.5, 157.7, 159.0 ppm; IR $\left(\mathrm{CHCl}_{3}\right): \bar{v}=3050,2953,1587,1468,1243,1106 \mathrm{~cm}^{-1} ; \mathrm{MS}\left(\mathrm{NH}_{4}^{+}\right): \mathrm{m} / \mathrm{z}: 245\left[\mathrm{M}+\mathrm{H}^{+}\right]$; elemental analysis calcd (\%) for $\mathrm{C}_{15} \mathrm{H}_{16} \mathrm{O}_{3}$ (244.29): C 73.75, H 6.60, O 19.65; found: C 73.66, H 6.62, O 19.59.

2,6,4'-Trimethoxybiphenyl (Table 4, entry 5): White solid, m.p. $122^{\circ} \mathrm{C} ; \mathrm{R}_{\mathrm{f}} 0.2$ (5\% AcOEt/hexane); GC retention time $11.83 \mathrm{~min} ;{ }^{1} \mathrm{H} \mathrm{NMR}\left(200 \mathrm{MHz},\left[\mathrm{D}_{1}\right] \mathrm{CHCl}_{3}, 20^{\circ} \mathrm{C}\right): 3.79\left(\mathrm{~s}, 6 \mathrm{H} ; 2 \mathrm{OCH}_{3}\right), 3.88\left(\mathrm{~s}, 3 \mathrm{H} ; \mathrm{OCH}_{3}\right)$, $6.69\left(\mathrm{~d},{ }^{3} \mathrm{~J}(\mathrm{H}, \mathrm{H}) 8.3 \mathrm{~Hz}, 2 \mathrm{H} ; 3,5-\mathrm{H}\right), 6.99\left(\mathrm{~d},{ }^{3} \mathrm{~J}(\mathrm{H}, \mathrm{H}) 9.0 \mathrm{~Hz}, 2 \mathrm{H} ; 3,5-\mathrm{H}\right), 7.26-7.35 \mathrm{ppm}(\mathrm{m}, 3 \mathrm{H} ; 4,2,6-\mathrm{H}) ;{ }^{13} \mathrm{C}$ $\operatorname{NMR}\left(75 \mathrm{MHz},\left[\mathrm{D}_{1}\right] \mathrm{CHCl}_{3}, 20^{\circ} \mathrm{C}\right): 55.0,55.8,104.2,113.2,119.1,126.1,128.3,131.9,157.7,158.2 \mathrm{ppm}$; IR $\left(\mathrm{CHCl}_{3}\right): \bar{v}=3015,2954,1588,1519,1471,1247,1112 \mathrm{~cm}^{-1} ; \mathrm{MS}\left(\mathrm{NH}_{4}{ }^{+}\right): \mathrm{m} / \mathrm{z}: 245\left[\mathrm{M}+\mathrm{H}^{+}\right]$; elemental analysis calcd (\%) for $\mathrm{C}_{15} \mathrm{H}_{16} \mathrm{O}_{3}$ (244.29): C 73.75, H 6.60, O 19.65; found: C 73.47, H 6.59, $\mathrm{O} 19.68$.

(2',6'-Dimethoxybiphenyl-3-yl)dimethylamine (Table 4, entries 6-8): White solid, m.p. $118^{\circ} \mathrm{C} ; \mathrm{R}_{\mathrm{f}} 0.3(30 \%$ $\left.\mathrm{Et}_{2} \mathrm{O} / \mathrm{hexane}\right) ; \mathrm{GC}$ retention time $12.30 \mathrm{~min} ;{ }^{1} \mathrm{H} \mathrm{NMR}\left(300 \mathrm{MHz},\left[\mathrm{D}_{1}\right] \mathrm{CHCl}_{3}, 20^{\circ} \mathrm{C}\right): 2.97(\mathrm{~s}, 6 \mathrm{H} ; \mathrm{NMe} 2)$, $3.76\left(\mathrm{~s}, 6 \mathrm{H} ; 2 \mathrm{OCH}_{3}\right), 6.68\left(\mathrm{~d},{ }^{3} \mathrm{~J}(\mathrm{H}, \mathrm{H}) 8.3 \mathrm{~Hz}, 2 \mathrm{H} ; 3,5-\mathrm{H}\right), 6.70-6.77(\mathrm{~m}, 3 \mathrm{H} ; 2,4,6-\mathrm{H}), 7.26$ - $7.33 \mathrm{ppm}(\mathrm{m}$, $2 \mathrm{H} ; 5,4-\mathrm{H}) ;{ }^{13} \mathrm{C} \mathrm{NMR}\left(50 \mathrm{MHz},\left[\mathrm{D}_{1}\right] \mathrm{CHCl}_{3}, 20^{\circ} \mathrm{C}\right): 40.6,55.8,104.1,111.3,115.4,119.3,120.4,128.2,128.3$, 134.7, 150.0, 157.7 ppm; IR $\left(\mathrm{CHCl}_{3}\right): \bar{v}=3054,2989,1604,1471,1266,1112 \mathrm{~cm}^{-1} ; \mathrm{MS}\left(\mathrm{NH}_{4}^{+}\right): \mathrm{m} / \mathrm{z}: 258$ $\left[\mathrm{M}+\mathrm{H}^{+}\right]$; elemental analysis calcd (\%) for $\mathrm{C}_{16} \mathrm{H}_{19} \mathrm{NO}_{2}$ (257.33): C 74.68, H 7.44, $\mathrm{O}$ 12.43; found: $\mathrm{C} 74.48, \mathrm{H}$ $7.45,012.46$.

2,6-Dimethoxy-3'-methylbiphenyl (Table 4, entries 9 - 14): Colorless oil; $R_{f} 0.5$ (10\% AcOEt/hexane); GC retention time $8.75 \mathrm{~min} ;{ }^{1} \mathrm{H} \mathrm{NMR}\left(300 \mathrm{MHz},\left[\mathrm{D}_{1}\right] \mathrm{CHCl}_{3}, 20^{\circ} \mathrm{C}\right): 2.41\left(\mathrm{~s}, 3 \mathrm{H} ; \mathrm{CH}_{3}\right), 3.76\left(\mathrm{~s}, 6 \mathrm{H} ; 2 \mathrm{OCH}_{3}\right), 6.67$ 
$\left(\mathrm{d},{ }^{3} \mathrm{~J}(\mathrm{H}, \mathrm{H}) 8.3 \mathrm{~Hz}, 2 \mathrm{H} ; 3,5-\mathrm{H}\right), 7.13-7.37 \mathrm{ppm}(\mathrm{m}, 5 \mathrm{H} ; 4-\mathrm{H}, 4 \mathrm{H}) ;{ }^{13} \mathrm{C} \mathrm{NMR}\left(75 \mathrm{MHz},\left[\mathrm{D}_{1}\right] \mathrm{CHCl}_{3}, 20^{\circ} \mathrm{C}\right): 21.6$, 56.1, 104.6, 120.3, 127.6, 127.7, 128.0, 128.6, 131.7, 134.2, 137.1, 158.0 ppm; IR $\left(\mathrm{CHCl}_{3}\right): \bar{v}=3048,2930$, $1606,1589,1466,1242,1110 \mathrm{~cm}^{-1}$; MS $\left(\mathrm{NH}_{4}^{+}\right): \mathrm{m} / \mathrm{z}: 229\left[\mathrm{M}+\mathrm{H}^{+}\right]$; elemental analysis calcd (\%) for $\mathrm{C}_{15} \mathrm{H}_{16} \mathrm{O}_{2}$ (228.29): C 78.92, H 7.06, O 14.02; found: C 78.71, H 7.05, 014.06.

2,6-Dimethoxy-4'-methylbiphenyl (Table 4, entries 12 - 14): White solid, m.p. $86^{\circ} \mathrm{C} ; R_{f} 0.5(10 \%$ AcOEt/hexane); $\mathrm{GC}$ retention time $9.15 \mathrm{~min} ;{ }^{1} \mathrm{H} \mathrm{NMR}\left(300 \mathrm{MHz},\left[\mathrm{D}_{1}\right] \mathrm{CHCl}_{3}, 20^{\circ} \mathrm{C}\right): 2.41\left(\mathrm{~s}, 3 \mathrm{H} ; \mathrm{CH}_{3}\right), 3.75$ $\left(\mathrm{s}, 6 \mathrm{H} ; 2 \mathrm{CCH}_{3}\right), 6.67\left(\mathrm{~d},{ }^{3} \mathrm{~J}(\mathrm{H}, \mathrm{H}) 8.3 \mathrm{~Hz}, 2 \mathrm{H} ; 3,5-\mathrm{H}\right), 7.21-7.32 \mathrm{ppm}(\mathrm{m}, 5 \mathrm{H} ; 4-\mathrm{H}, 4 \mathrm{H}) ;{ }^{13} \mathrm{C} \mathrm{NMR}\left(75 \mathrm{MHz},\left[\mathrm{D}_{1}\right]\right.$ $\left.\mathrm{CHCl}_{3}, 20^{\circ} \mathrm{C}\right): 21.4,56.1,104.7,120.1,128.6,128.7,130.9,131.3,136.3,158.1 \mathrm{ppm} ; \mathrm{IR}\left(\mathrm{CHCl}_{3}\right): \bar{v}=3053$, 2986, 1653, 1589, 1469, 1264, $1110 \mathrm{~cm}^{-1}$; MS $\left(\mathrm{NH}_{4}{ }^{+}\right): \mathrm{m} / \mathrm{z}: 229\left[\mathrm{M}+\mathrm{H}^{+}\right]$; elemental analysis calcd (\%) for $\mathrm{C}_{15} \mathrm{H}_{16} \mathrm{O}_{2}$ (228.29): C 78.92, H 7.06, O 14.02; found: C 78.71, H 7.04, O 13.98.

2,6-Dimethoxy-4'-trifluoromethylbiphenyl (Table 4, entries 15, 16): White solid, m.p. $82^{\circ} \mathrm{C} ; R_{f} 0.5(10 \%$ AcOEt/hexane); $\mathrm{GC}$ retention time $7.71 \mathrm{~min} ;{ }^{1} \mathrm{H} \mathrm{NMR}\left(200 \mathrm{MHz},\left[\mathrm{D}_{1}\right] \mathrm{CHCl}_{3}, 20^{\circ} \mathrm{C}\right): 3.76\left(\mathrm{~s}, 6 \mathrm{H} ; 2 \mathrm{OCH}_{3}\right)$, $6.68\left(\mathrm{~d},{ }^{3} \mathrm{~J}(\mathrm{H}, \mathrm{H}) 8.3 \mathrm{~Hz}, 2 \mathrm{H} ; 3,5-\mathrm{H}\right), 7.38\left(\mathrm{t},{ }^{3} \mathrm{~J}(\mathrm{H}, \mathrm{H}) 8.3 \mathrm{~Hz}, 1 \mathrm{H} ; 4-\mathrm{H}\right), 7.48\left(\mathrm{~d},{ }^{3} \mathrm{~J}(\mathrm{H}, \mathrm{H}) 8.0 \mathrm{~Hz}, 2 \mathrm{H} ; 2,6-\mathrm{H}\right), 7.66$ $\operatorname{ppm}\left(\mathrm{d},{ }^{3} \mathrm{~J}(\mathrm{H}, \mathrm{H}) 8.3 \mathrm{~Hz}, 2 \mathrm{H} ; 3,5-\mathrm{H}\right) ;{ }^{13} \mathrm{C} \mathrm{NMR}\left(75 \mathrm{MHz},\left[\mathrm{D}_{1}\right] \mathrm{CHCl}_{3}, 20^{\circ} \mathrm{C}\right): 55.8,104.2,118.0,124.5,124.5$ (q, $\left.{ }^{1} \mathrm{~J}(\mathrm{C}, \mathrm{F}) 270 \mathrm{~Hz}\right), 127.9,129.4,131.4,138.1,157.5$ ppm; IR $\left(\mathrm{CHCl}_{3}\right): \bar{v}=3059,2924,1664,1592,1469$, 1248, $1113 \mathrm{~cm}^{-1}$; $\mathrm{MS}\left(\mathrm{NH}_{4}^{+}\right): \mathrm{m} / \mathrm{z}: 300\left[\mathrm{M}+\mathrm{NH}_{4}^{+}\right]$; elemental analysis calcd (\%) for $\mathrm{C}_{15} \mathrm{H}_{13} \mathrm{~F}_{3} \mathrm{O}_{2}$ (282.26): $\mathrm{C}$ 63.83, H 4.64, O 11.34; found: C 64.01, H 4.63, O 11.30.

2,6-Dimethoxy-3'-trifluoromethylbiphenyl (Table 4, entries 15, 16): Colorless oil; $R_{f} 0.5$ (10\% AcOEt/hexane); $\mathrm{GC}$ retention time $7.37 \mathrm{~min} ;{ }^{1} \mathrm{H} \mathrm{NMR}\left(200 \mathrm{MHz},\left[\mathrm{D}_{1}\right] \mathrm{CHCl}_{3}, 20^{\circ} \mathrm{C}\right): 3.81\left(\mathrm{~s}, 6 \mathrm{H} ; 2 \mathrm{OCH}_{3}\right)$, $6.73\left(\mathrm{~d},{ }^{3} \mathrm{~J}(\mathrm{H}, \mathrm{H}) 8.3 \mathrm{~Hz}, 2 \mathrm{H} ; 3,5-\mathrm{H}\right), 7.34\left(\mathrm{t},{ }^{3} \mathrm{~J}(\mathrm{H}, \mathrm{H}) 8.4 \mathrm{~Hz}, 1 \mathrm{H} ; 4-\mathrm{H}\right), 7.51-7.78 \mathrm{ppm}(\mathrm{m}, 4 \mathrm{H} ; 4 \mathrm{H}) ;{ }^{13} \mathrm{C} \mathrm{NMR}$ (75 MHz, [ $\left.\mathrm{D}_{1}\right] \mathrm{CHCl}_{3}, 20^{\circ} \mathrm{C}$ ): 56.3, 104.6, 118.3, 124.0, 125.0 (q, ${ }^{1} \mathrm{~J}(\mathrm{C}, \mathrm{F}) 270 \mathrm{~Hz}$ ), 128.5, 129.8, 130.0, 130.7, 134.9, 135.3, 157.9 ppm; IR $\left(\mathrm{CH}_{2} \mathrm{Cl}_{2}\right): \bar{v}=3011,2955,1589,1467,1239,1111 \mathrm{~cm}^{-1} ; \mathrm{MS}\left(\mathrm{NH}_{4}^{+}\right): \mathrm{m} / \mathrm{z}: 300$ $\left[\mathrm{M}+\mathrm{NH}_{4}{ }^{+}\right]$; elemental analysis calcd (\%) for $\mathrm{C}_{15} \mathrm{H}_{13} \mathrm{~F}_{3} \mathrm{O}_{2}$ (282.26): C 63.83, H 4.64, O 11.34; found: C 63.75, H 4.63, O 11.31 .

Biphenyl-3-yldimethylamine: Colorless oil; $\mathrm{R}_{\mathrm{f}} 0.6\left(10 \% \mathrm{Et}_{2} \mathrm{O} /\right.$ hexane $) ;{ }^{1} \mathrm{H} \mathrm{NMR}\left(200 \mathrm{MHz},\left[\mathrm{D}_{1}\right] \mathrm{CHCl}_{3}\right.$, $\left.20^{\circ} \mathrm{C}\right): 3.08\left(\mathrm{~s}, 6 \mathrm{H} ; \mathrm{NMe}_{2}\right), 6.75-7.08(\mathrm{~m}, 3 \mathrm{H} ; 2,4,6-\mathrm{H}), 7.32-7.69 \mathrm{ppm}(\mathrm{m}, 6 \mathrm{H} ; 5-\mathrm{H}, 5 \mathrm{H}) ;{ }^{13} \mathrm{C} \mathrm{NMR}(75 \mathrm{MHz}$, $\left.\left[\mathrm{D}_{1}\right] \mathrm{CHCl}_{3}, 20^{\circ} \mathrm{C}\right): 40.6,111.5,111.6,115.8,127.0,127.3,128.5,129.4,142.2,150.9 \mathrm{ppm} ; \mathrm{IR}\left(\mathrm{CHCl}_{3}\right)$ : $\bar{v}=3008,2884,1601,1568,1487,1214 \mathrm{~cm}^{-1} ; \mathrm{MS}\left(\mathrm{NH}_{4}{ }^{+}\right): \mathrm{m} / \mathrm{z}: 198\left[\mathrm{M}+\mathrm{H}^{+}\right]$; elemental analysis calcd (\%) for $\mathrm{C}_{14} \mathrm{H}_{15} \mathrm{~N}$ (197.28): C 85.24, H 7.66; found: C 85.51, H 7.65.

3-Methoxybiphenyl: Colorless oil; $\mathrm{R}_{\mathrm{f}} 0.3$ (10\% AcOEt/hexane); GC retention time $7.15 \mathrm{~min}$; ${ }^{1} \mathrm{H}$ NMR (300 $\left.\mathrm{MHz},\left[\mathrm{D}_{1}\right] \mathrm{CHCl}_{3}, 20^{\circ} \mathrm{C}\right): 3.90\left(\mathrm{~s}, 3 \mathrm{H} ; \mathrm{OCH}_{3}\right), 6.93\left(\mathrm{dd},{ }^{3} \mathrm{~J}(\mathrm{H}, \mathrm{H}) 8.3 \mathrm{~Hz},{ }^{4} \mathrm{~J}(\mathrm{H}, \mathrm{H}) 5.5 \mathrm{~Hz}, 2 \mathrm{H} ; 2-\mathrm{H}\right), 7.17$ - 7.26 $(\mathrm{m}, 2 \mathrm{H} ; 4,6-\mathrm{H}), 7.38-7.66 \mathrm{ppm}(\mathrm{m}, 6 \mathrm{H}, 5-\mathrm{H} ; 5 \mathrm{H}) ;{ }^{13} \mathrm{C} \mathrm{NMR}\left(75 \mathrm{MHz},\left[\mathrm{D}_{1}\right] \mathrm{CHCl}_{3}, 20^{\circ} \mathrm{C}\right): 55.2,112.6,112.9$, $119.7,127.2,127.4,128.7,129.7,141.1,142.7,159.9$ ppm; IR $\left(\mathrm{CHCl}_{3}\right): \bar{v}=3054,2959,1599,1479,1266$, $1220 \mathrm{~cm}^{-1}$; MS $\left(\mathrm{NH}_{4}^{+}\right): \mathrm{m} / \mathrm{z}: 185\left[\mathrm{M}+\mathrm{H}^{+}\right]$; elemental analysis calcd (\%) for $\mathrm{C}_{13} \mathrm{H}_{12} \mathrm{O}$ (184.24): $\mathrm{C} 84.75, \mathrm{H} \mathrm{6.56}$, O 8.68; found: C 84.52, H 6.58, 08.65 .

2,6,3',5'-Tetramethoxybiphenyl (Table 5, entry 1): White solid, m.p. $122^{\circ} \mathrm{C} ; \mathrm{R}_{\mathrm{f}} 0.3$ (10\% AcOEt/hexane); $\mathrm{GC}$ retention time $17.23 \mathrm{~min} ;{ }^{1} \mathrm{H} \mathrm{NMR}\left(200 \mathrm{MHz},\left[\mathrm{D}_{1}\right] \mathrm{CHCl}_{3}, 20^{\circ} \mathrm{C}\right): 3.79\left(\mathrm{~s}, 6 \mathrm{H} ; 2 \mathrm{OCH}_{3}\right), 3.84\left(\mathrm{~s}, 6 \mathrm{H} ; 2 \mathrm{OCH}_{3}\right)$, $6.50\left(\mathrm{t},{ }^{4} \mathrm{~J}(\mathrm{H}, \mathrm{H}) 2.2 \mathrm{~Hz}, 1 \mathrm{H} ; 4-\mathrm{H}\right), 6.55\left(\mathrm{~d},{ }^{4} \mathrm{~J}(\mathrm{H}, \mathrm{H}) 2.2 \mathrm{~Hz}, 2 \mathrm{H} ; 2,6-\mathrm{H}\right), 6.69\left(\mathrm{~d},{ }^{3} \mathrm{~J}(\mathrm{H}, \mathrm{H}) 8.3 \mathrm{~Hz}, 2 \mathrm{H} ; 3,5-\mathrm{H}\right), 7.32$ ppm $\left(\mathrm{t},{ }^{3} \mathrm{~J}(\mathrm{H}, \mathrm{H}) 8.3 \mathrm{~Hz}, 1 \mathrm{H} ; 4-\mathrm{H}\right) ;{ }^{13} \mathrm{C} \mathrm{NMR}\left(50 \mathrm{MHz},\left[\mathrm{D}_{1}\right] \mathrm{CHCl}_{3}, 20^{\circ} \mathrm{C}\right): 55.7,56.4,99.7,104.6,109.4,119.9$, 129.2, 136.5, 158.1, 160.5 ppm; IR $\left(\mathrm{CHCl}_{3}\right): \bar{v}=3094,2944,1598,1450,1253,1194,1149 \mathrm{~cm}^{-1}$; $\mathrm{MS}_{\left(\mathrm{NH}_{4}^{+}\right):}$ 
m/z: $275\left[\mathrm{M}+\mathrm{H}^{+}\right]$; elemental analysis calcd (\%) for $\mathrm{C}_{16} \mathrm{H}_{18} \mathrm{O}_{4}$ (274.31): C 70.06, H 6.61, O 23.33; found: C 69.81, H 6.63, O 23.41.

2,6,3',4'-Tetramethoxybiphenyl (Table 5, entry 2): White needles, m.p. $148^{\circ} \mathrm{C} ; \mathrm{R}_{\mathrm{f}} 0.5$ (30\% Et $\mathrm{H}_{2} \mathrm{O} /$ hexane); ${ }^{1} \mathrm{H} \mathrm{NMR}\left(200 \mathrm{MHz},\left[\mathrm{D}_{1}\right] \mathrm{CHCl}_{3}, 20^{\circ} \mathrm{C}\right): 3.79(\mathrm{~s}, 6 \mathrm{H} ; 2 \mathrm{OCH}), 3.90\left(\mathrm{~s}, 3 \mathrm{H} ; \mathrm{OCH}_{3}\right), 3.96\left(\mathrm{~s}, 3 \mathrm{H} ; \mathrm{OCH}_{3}\right), 6.70(\mathrm{~d}$, $\left.{ }^{3} \mathrm{~J}(\mathrm{H}, \mathrm{H}) 8.3 \mathrm{~Hz}, 2 \mathrm{H} ; 3,5-\mathrm{H}\right), 6.93-6.99(\mathrm{~m}, 3 \mathrm{H} ; 2,5,6-\mathrm{H}), 7.29 \mathrm{ppm}\left(\mathrm{t},{ }^{3} \mathrm{~J}(\mathrm{H}, \mathrm{H}) 8.3 \mathrm{~Hz}, 1 \mathrm{H} ; 4-\mathrm{H}\right) ;{ }^{13} \mathrm{C}$ NMR $(50$ $\left.\mathrm{MHz},\left[\mathrm{D}_{1}\right] \mathrm{CHCl}_{3}, 20^{\circ} \mathrm{C}\right): 55.7,55.8,55.9,104.2,110.5,114.3,119.2,123.1,126.4,128.4,147.7,148.1,157.8$ ppm; IR $\left(\mathrm{CHCl}_{3}\right): \bar{v}=3012,2935,1587,1523,1470,1292,1247,1106,1024 \mathrm{~cm}^{-1} ; \mathrm{MS}\left(\mathrm{NH}_{4}^{+}\right): \mathrm{m} / \mathrm{z}: 275[\mathrm{MH}] ;$ elemental analysis calcd (\%) for $\mathrm{C}_{16} \mathrm{H}_{18} \mathrm{O}_{4}$ (274.31): C 70.06, H 6.61, O 23.33; found: C 70.31, H 6.62, O 23.39.

5-(2,6-Dimethoxyphenyl)benzo[1,3]dioxole (Table 5, entry 3): White solid, m.p. $90^{\circ} \mathrm{C} ; \mathrm{R}_{\mathrm{f}} 0.2$ (5\% Et ${ }_{2} \mathrm{O}$ /hexane); ${ }^{1} \mathrm{H} N M R\left(300 \mathrm{MHz},\left[\mathrm{D}_{1}\right] \mathrm{CHCl}_{3}, 20^{\circ} \mathrm{C}\right): 3.76\left(\mathrm{~s}, 6 \mathrm{H} ; 2 \mathrm{OCH}_{3}\right), 5.99\left(\mathrm{~s}, 2 \mathrm{H} ; \mathrm{OCH}_{2} \mathrm{O}\right), 6.65\left(\mathrm{~d},{ }^{3} \mathrm{~J}(\mathrm{H}, \mathrm{H})\right.$ $8.1 \mathrm{~Hz}, 2 \mathrm{H} ; 3,5-\mathrm{H}), 6.80-6.90(\mathrm{~m}, 3 \mathrm{H} ; 3,4,6-\mathrm{H}), 7.27 \mathrm{ppm}\left(\mathrm{t},{ }^{3} \mathrm{~J}(\mathrm{H}, \mathrm{H}) 8.1 \mathrm{~Hz}, 1 \mathrm{H} ; 4-\mathrm{H}\right) ;{ }^{13} \mathrm{C} \mathrm{NMR}(75 \mathrm{MHz}$, $\left.\left[\mathrm{D}_{1}\right] \mathrm{CHCl}_{3}, 20^{\circ} \mathrm{C}\right): 55.9,100.8,104.2,107.9,111.5,119.1,124.2,127.5,128.5,146.3,147.0,157.8 \mathrm{ppm}$; IR $\left(\mathrm{CHCl}_{3}\right): \bar{v}=3025,2997,1590,1500,1467,1214 \mathrm{~cm}^{-1} ; \mathrm{MS}\left(\mathrm{NH}_{4}^{+}\right): \mathrm{m} / \mathrm{z}: 276\left[\mathrm{M}+\mathrm{NH}_{4}{ }^{+}\right]$; elemental analysis calcd (\%) for $\mathrm{C}_{15} \mathrm{H}_{14} \mathrm{O}_{4}$ (258.27): C 69.76, H 5.46, O 24.78; found: C 70.01, H 5.48, O 24.84.

2,2',6'-Trimethoxy-5-methylbiphenyl (Table 5, entry 4): White solid, m.p. $114^{\circ} \mathrm{C} ; \mathrm{R}_{\mathrm{f}} 0.3$ (20\% Et $\mathrm{t}_{2} \mathrm{O}$ /hexane); ${ }^{1} \mathrm{H} \mathrm{NMR}\left(300 \mathrm{MHz},\left[\mathrm{D}_{1}\right] \mathrm{CHCl}_{3}, 20^{\circ} \mathrm{C}\right): 2.37\left(\mathrm{~s}, 3 \mathrm{H} ; \mathrm{CH}_{3}\right), 3.77\left(\mathrm{~s}, 3 \mathrm{H} ; \mathrm{OCH}_{3}\right), 3.78\left(\mathrm{~s}, 6 \mathrm{H} ; 2 \mathrm{OCH}_{3}\right)$, $6.69\left(\mathrm{~d},{ }^{3} \mathrm{~J}(\mathrm{H}, \mathrm{H}) 8.3 \mathrm{~Hz}, 2 \mathrm{H} ; 3,5-\mathrm{H}\right), 6.93\left(\mathrm{~d},{ }^{3} \mathrm{~J}(\mathrm{H}, \mathrm{H}) 8.3 \mathrm{~Hz}, 1 \mathrm{H} ; 3-\mathrm{H}\right), 7.04\left(\mathrm{~d},{ }^{4} \mathrm{~J}(\mathrm{H}, \mathrm{H}) 1.9 \mathrm{~Hz}, 1 \mathrm{H} ; 6-\mathrm{H}\right), 7.16$ (dd, $\left.{ }^{3} \mathrm{~J}(\mathrm{H}, \mathrm{H}) 8.3 \mathrm{~Hz},{ }^{4} \mathrm{~J}(\mathrm{H}, \mathrm{H}) 1.9 \mathrm{~Hz}, 1 \mathrm{H} ; 4-\mathrm{H}\right), 7.32 \mathrm{ppm}\left(\mathrm{t},{ }^{3} \mathrm{~J}(\mathrm{H}, \mathrm{H}) 8.3 \mathrm{~Hz}, 1 \mathrm{H} ; 4-\mathrm{H}\right) ;{ }^{13} \mathrm{C} \mathrm{NMR}\left(75 \mathrm{MHz},\left[\mathrm{D}_{1}\right]\right.$ $\left.\mathrm{CHCl}_{3}, 20^{\circ} \mathrm{C}\right): 18.7,55.2,55.9,104.0,112.8,116.2,118.9,128.7,129.4,130.2,157.1,157.7 \mathrm{ppm} ; \mathrm{IR}\left(\mathrm{CHCl}_{3}\right)$ : $\bar{v}=2991,2931,1607,1586,1507,1250,1109,1040 \mathrm{~cm}^{-1} ; \mathrm{MS}\left(\mathrm{NH}_{4}^{+}\right): \mathrm{m} / \mathrm{z}: 259\left[\mathrm{M}+\mathrm{H}^{+}\right]$; elemental analysis calcd (\%) for $\mathrm{C}_{16} \mathrm{H}_{18} \mathrm{O}_{3}$ (258.32): C 74.39, H 7.02, O 18.58; found: C 74.67, H 7.04, O 18.52.

5,2',6'-Trimethoxy-2-methylbiphenyl (Table 5, entry 5): White solid, m.p. $99^{\circ} \mathrm{C} ; \mathrm{R}_{\mathrm{f}} 0.2\left(20 \% \mathrm{Et}_{2} \mathrm{O}\right.$ /hexane); ${ }^{1} \mathrm{H} \mathrm{NMR}\left(300 \mathrm{MHz},\left[\mathrm{D}_{1}\right] \mathrm{CHCl}_{3}, 20^{\circ} \mathrm{C}\right): 2.04$ (s, 3H; $\left.\mathrm{CH}_{3}\right), 3.77\left(\mathrm{~s}, 6 \mathrm{H} ; 2 \mathrm{OCH}_{3}\right), 3.83\left(\mathrm{~s}, 3 \mathrm{H} ; \mathrm{OCH}_{3}\right)$, $6.69\left(\mathrm{~d},{ }^{3} \mathrm{~J}(\mathrm{H}, \mathrm{H}) 8.3 \mathrm{~Hz}, 2 \mathrm{H} ; 3,5-\mathrm{H}\right), 6.76\left(\mathrm{~d},{ }^{4} \mathrm{~J}(\mathrm{H}, \mathrm{H}) 2.9 \mathrm{~Hz}, 1 \mathrm{H} ; 6-\mathrm{H}\right), 6.86\left(\mathrm{dd},{ }^{3} \mathrm{~J}(\mathrm{H}, \mathrm{H}) 8.3 \mathrm{~Hz},{ }^{4} \mathrm{~J}(\mathrm{H}, \mathrm{H}) 2.9\right.$ $\mathrm{Hz}, 1 \mathrm{H} ; 4-\mathrm{H}), 7.22\left(\mathrm{~d},{ }^{3} \mathrm{~J}(\mathrm{H}, \mathrm{H}) 8.3 \mathrm{~Hz}, 1 \mathrm{H} ; 3-\mathrm{H}\right), 7.35 \mathrm{ppm}\left(\mathrm{t},{ }^{3} \mathrm{~J}(\mathrm{H}, \mathrm{H}) 8.3 \mathrm{~Hz}, 1 \mathrm{H} ; 4-\mathrm{H}\right) ;{ }^{13} \mathrm{C} \mathrm{NMR}\left(75 \mathrm{MHz},\left[\mathrm{D}_{1}\right]\right.$ $\left.\mathrm{CHCl}_{3}, 20^{\circ} \mathrm{C}\right): 20.5,55.9,104.1,111.2,116.3,123.2,128.5,128.9,132.6,155.4,158.0 \mathrm{ppm}$; IR $\left(\mathrm{CHCl}_{3}\right):$ $\bar{v}=3005,2935,1607,1590,1500,1468,1246,1111 \mathrm{~cm}^{-1} ; \mathrm{MS}\left(\mathrm{NH}_{4}^{+}\right): \mathrm{m} / \mathrm{z}: 259\left[\mathrm{M}+\mathrm{H}^{+}\right]$; elemental analysis calcd (\%) for $\mathrm{C}_{16} \mathrm{H}_{18} \mathrm{O}_{3}$ (258.32): C 74.39, H 7.02, O 18.58; found: C 74.21, H 7.01, O 18.61.

1-(2,6-Dimethoxyphenyl)naphthalene: White solid, m.p. $108^{\circ} \mathrm{C} ; \mathrm{R}_{\mathrm{f}} 0.3$ (4\% AcOEt/hexane); ${ }^{1} \mathrm{H}$ NMR (200 $\left.\mathrm{MHz},\left[\mathrm{D}_{1}\right] \mathrm{CHCl}_{3}, 20^{\circ} \mathrm{C}\right): 3.69\left(\mathrm{~s}, 6 \mathrm{H} ; 2 \mathrm{OCH}_{3}\right), 6.76\left(\mathrm{~d},{ }^{3} \mathrm{~J}(\mathrm{H}, \mathrm{H}) 8.3 \mathrm{~Hz}, 2 \mathrm{H} ; 3,5-\mathrm{H}\right), 7.34$ - $7.54(\mathrm{~m}, 6 \mathrm{H}$; naphthalene $\mathrm{H}), 7.59\left(\mathrm{t},{ }^{3} \mathrm{~J}(\mathrm{H}, \mathrm{H}) \quad 8.3 \mathrm{~Hz}, 1 \mathrm{H} ; 4-\mathrm{H}\right), 7.88$ - $7.98 \mathrm{ppm}(\mathrm{m}, 2 \mathrm{H}$; naphthalene $\mathrm{H}) ;{ }^{13} \mathrm{C}$ NMR $(75$ $\left.\mathrm{MHz},\left[\mathrm{D}_{1}\right] \mathrm{CHCl}_{3}, 20^{\circ} \mathrm{C}\right): 55.9,104.2,125.3$ 125.4, 126.0, 127.4, 128.0, 128.1, 129.1, 132.6, 133.5, 158.4 ppm; IR $\left(\mathrm{CHCl}_{3}\right): \bar{v}=3025,2952,1596,1529,1472,1214,1113 \mathrm{~cm}^{-1} ; \mathrm{MS}\left(\mathrm{NH}_{4}^{+}\right): \mathrm{m} / \mathrm{z}: 265\left[\mathrm{M}+\mathrm{H}^{+}\right]$; elemental analysis calcd (\%) for $\mathrm{C}_{18} \mathrm{H}_{16} \mathrm{O}_{2}$ (264.32): C 81.79, $\mathrm{H}$ 6.10, $\mathrm{O}$ 12.11; found: C 82.01, H 6.08, $\mathrm{O} 12.15$.

2-(2,6-Dimethoxyphenyl)naphthalene: White solid, m.p. $83^{\circ} \mathrm{C} ; \mathrm{R}_{\mathrm{f}} 0.3$ (4\% AcOEt/hexane); ${ }^{1} \mathrm{H}$ NMR (200 $\left.\mathrm{MHz},\left[\mathrm{D}_{1}\right] \mathrm{CHCl}_{3}, 20^{\circ} \mathrm{C}\right): 3.80\left(\mathrm{~s}, 6 \mathrm{H} ; 2 \mathrm{CH}_{3}\right), 6.75\left(\mathrm{~d},{ }^{3} \mathrm{~J}(\mathrm{H}, \mathrm{H}) 8.3 \mathrm{~Hz}, 2 \mathrm{H} ; 3,5-\mathrm{H}\right), 7.38\left(\mathrm{t},{ }^{3} \mathrm{~J}(\mathrm{H}, \mathrm{H}) 8.3 \mathrm{~Hz}, 1 \mathrm{H}\right.$; 4-H), 7.46 - 7.57 (m, 3H; naphthalene $H), 7.84-8.00$ ppm (m, 4H; naphthalene $H)$; ${ }^{13} \mathrm{C} \mathrm{NMR} \mathrm{(75} \mathrm{MHz,}\left[\mathrm{D}_{1}\right]$ $\left.\mathrm{CHCl}_{3}, 20^{\circ} \mathrm{C}\right): 55.9,104.3,119.5,125.5,126.9,127.7,128.1,128.8,129.3,129.7,131.7,132.5,133.3,157.9$ ppm; IR $\left(\mathrm{CHCl}_{3}\right): \bar{v}=3003,2941,1585,1496,1274,1105 \mathrm{~cm}^{-1} ; \mathrm{MS}\left(\mathrm{NH}_{4}^{+}\right): \mathrm{m} / \mathrm{z}: 265\left[\mathrm{M}+\mathrm{H}^{+}\right]$; elemental analysis calcd (\%) for $\mathrm{C}_{18} \mathrm{H}_{16} \mathrm{O}_{2}$ (264.32): C 81.79, $\mathrm{H}$ 6.10, $\mathrm{O}$ 12.11; found: $\mathrm{C} 81.97, \mathrm{H} 6.11, \mathrm{O} 12.09$. 
2-Phenylthiophene (Scheme 1): A solution of n-butyllithium (1.6M solution in hexane, $6.9 \mathrm{~mL}, 11 \mathrm{mmol}$ ) was added dropwise at $40^{\circ} \mathrm{C}$ to a solution of thiophene $(11 \mathrm{mmol})$ in THF $(8.2 \mathrm{~mL})$. The mixture was stirred for $1 \mathrm{~h}$ at $30^{\circ} \mathrm{C}$, then cooled at $40^{\circ} \mathrm{C}$, and a solution of fluorobenzene $(5 \mathrm{mmol})$ in THF $(3.2 \mathrm{~mL})$ was added dropwise. The resulting mixture was warmed to RT for $1 \mathrm{~h}$ and heated to $60^{\circ} \mathrm{C}$ for $12 \mathrm{~h}$. The reaction mixture was allowed to cool to RT and quenched by addition of water $(40 \mathrm{~mL})$. The aqueous layer was extracted with $\mathrm{Et}_{2} \mathrm{O}(60 \mathrm{~mL})$. The organic phase was dried over $\mathrm{MgSO}_{4}$, filtered, and concentrated under vacuum. The residue was purified by flash chromatography on silica gel using hexane as eluent. Yield 552 $\mathrm{mg}(69 \%)$ of white solid, m.p. $35^{\circ} \mathrm{C} ; \mathrm{R}_{\mathrm{f}} 0.5$ (hexane); ${ }^{1} \mathrm{H} \mathrm{NMR}\left(300 \mathrm{MHz},\left[\mathrm{D}_{1}\right] \mathrm{CHCl}_{3}, 20^{\circ} \mathrm{C}\right): 7.11-7.16$ (m, $\left.1 \mathrm{H} ; 5-\mathrm{H}), 7.31-7.70 \mathrm{ppm}(\mathrm{m}, 7 \mathrm{H} ; 3,4-\mathrm{H}, 5 \mathrm{H}) ;{ }^{13} \mathrm{C} \mathrm{NMR} \mathrm{(50} \mathrm{MHz},\left[\mathrm{D}_{1}\right] \mathrm{CHCl}_{3}, 20^{\circ} \mathrm{C}\right): 123.0,124.8,125.9$,

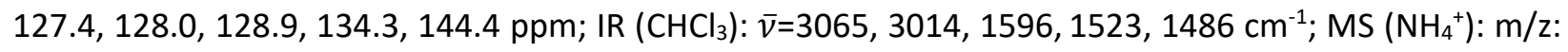
$161\left[\mathrm{M}+\mathrm{H}^{+}\right]$; elemental analysis calcd (\%) for $\mathrm{C}_{10} \mathrm{H}_{8} \mathrm{~S}$ (160.24): C 74.96, H 5.03; found: C 74.68, H 5.04.

2-Phenylbenzofuran (Scheme 1): A solution of n-butyllithium (1.6M solution in hexane, $3.3 \mathrm{~mL}, 5.3 \mathrm{mmol}$ ) was added dropwise at $10^{\circ} \mathrm{C}$ to a solution of benzofuran $(5.3 \mathrm{mmol})$ in THF $(10 \mathrm{~mL})$. The reaction mixture was stirred at $10^{\circ} \mathrm{C}$ for $1 \mathrm{~h}$ and warmed to $0^{\circ} \mathrm{C}$; then a solution of fluorobenzene $(2.4 \mathrm{mmol})$ in THF (1.6 $\mathrm{mL}$ ) was added dropwise. After the reaction mixture had been stirred for $1 \mathrm{~h}$ at $\mathrm{RT}$, it was heated to $60^{\circ} \mathrm{C}$ for $12 \mathrm{~h}$, then allowed to cool to RT, and quenched by addition of water $(40 \mathrm{~mL})$. The aqueous layer was extracted with $\mathrm{Et}_{2} \mathrm{O}(50 \mathrm{~mL})$, dried over $\mathrm{MgSO}_{4}$, and concentrated under vacuum. The residue was purified by flash chromatography on silica gel using hexane as eluent. Yield $102 \mathrm{mg}$ (22\%) of a white solid, m.p. $123^{\circ} \mathrm{C} ; R_{\mathrm{f}} 0.3$ (hexane); ${ }^{1} \mathrm{H} N M R\left(200 \mathrm{MHz},\left[\mathrm{D}_{1}\right] \mathrm{CHCl}_{3}, 20^{\circ} \mathrm{C}\right): 7.08(\mathrm{~s}, 1 \mathrm{H} ; 3-\mathrm{H}), 7.20-7.70(\mathrm{~m}, 8 \mathrm{H}), 7.88-$ $7.97 \mathrm{ppm}(\mathrm{m}, 2 \mathrm{H} ; 2 \mathrm{H}) ;{ }^{13} \mathrm{C} \mathrm{NMR}\left(75 \mathrm{MHz},\left[\mathrm{D}_{1}\right] \mathrm{CHCl}_{3}, 20^{\circ} \mathrm{C}\right): 101.3,111.2,120.9,122.9,124.2,124.9,128.5$, 128.8, 129.2, 130.5, 154.9, 155.9 ppm; IR $\left(\mathrm{CHCl}_{3}\right): \bar{v}=3020,1615,1510,1492 \mathrm{~cm}^{-1} ; \mathrm{MS}\left(\mathrm{NH}_{4}^{+}\right): \mathrm{m} / \mathrm{z}: 195$ $\left[\mathrm{M}+\mathrm{H}^{+}\right]$; elemental analysis calcd (\%) for $\mathrm{C}_{14} \mathrm{H}_{10} \mathrm{O}$ (194.23): $\mathrm{C} 86.57, \mathrm{H} 5.19, \mathrm{O} 8.24$; found: $\mathrm{C} 86.81, \mathrm{H}$ 5.17, O 8.26.

2-Chloro-1-methoxy-3-deuterobenzene: Colorless liquid; $\mathrm{R}_{\mathrm{f}} 0.5$ (hexane); ${ }^{1} \mathrm{H} \mathrm{NMR} \mathrm{(300} \mathrm{MHz},\left[\mathrm{D}_{1}\right] \mathrm{CHCl}_{3}$, $\left.20^{\circ} \mathrm{C}\right): 3.92\left(\mathrm{~s}, 3 \mathrm{H} ; \mathrm{OCH}_{3}\right), 6.92(\mathrm{~m}, 2 \mathrm{H} ; 4,6-\mathrm{H}), 7.24 \mathrm{ppm}\left(\mathrm{t},{ }^{3} \mathrm{~J}(\mathrm{H}, \mathrm{H}) 7.8 \mathrm{~Hz}, 1 \mathrm{H} ; 5-\mathrm{H}\right) ;{ }^{13} \mathrm{C} \mathrm{NMR}(75 \mathrm{MHz}$, $\left.\left[\mathrm{D}_{1}\right] \mathrm{CHCl} 3,20^{\circ} \mathrm{C}\right): 56.0,112.0,121.1,122.3,127.7,129.9\left(\mathrm{t},{ }^{1} \mathrm{~J}(\mathrm{C}, \mathrm{H}) 24.5 \mathrm{~Hz}, \mathrm{C}, \mathrm{D}\right), 155.0 \mathrm{ppm}$; IR $\left(\mathrm{CHCl}_{3}\right)$ : $\bar{v}=3069,2922,1583,1474,1434,1272,1047 \mathrm{~cm}^{-1} ; \mathrm{MS}\left(\mathrm{NH}_{4}{ }^{+}\right): \mathrm{m} / \mathrm{z}: 145\left[\mathrm{M}+\mathrm{H}^{+}\right]$; elemental analysis calcd (\%) for $\mathrm{C}_{7} \mathrm{H}_{6} \mathrm{ClOD}$ (143.58): C 58.56, H 4.21, O 11.14; found: C 58.37, H 4.22, $\mathrm{O} 11.10$.

2,6,2'-Trimethoxy-6'-deuterobiphenyl (Scheme 4): White solid, m.p. $140^{\circ} \mathrm{C} ; \mathrm{R}_{\mathrm{f}} 0.2$ (10\% AcOEt/hexane); ${ }^{1} \mathrm{H}$ NMR $\left(300 \mathrm{MHz},\left[\mathrm{D}_{3}\right] \mathrm{CH}_{3} \mathrm{CN}, 20^{\circ} \mathrm{C}\right): 3.68\left(\mathrm{~s}, 6 \mathrm{H} ; 2 \mathrm{OCH}_{3}\right), 3.70\left(\mathrm{~s}, 3 \mathrm{H} ; \mathrm{OCH}_{3}\right), 6.70\left(\mathrm{~d},{ }^{3} \mathrm{~J}(\mathrm{H}, \mathrm{H}) 8.1 \mathrm{~Hz}, 2 \mathrm{H}\right.$;

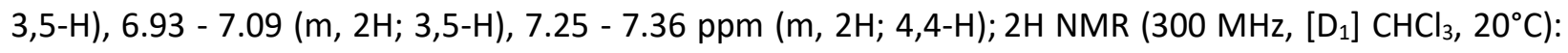
7.40 ppm (6-D); $\left.{ }^{13} \mathrm{C} \mathrm{NMR} \mathrm{(125} \mathrm{MHz,}\left[\mathrm{D}_{3}\right] \mathrm{CH}_{3} \mathrm{CN}, 20^{\circ} \mathrm{C}\right)$ 56.2, 56.4, 105.2, 112.1, 112.2, 121.0, 124.0 (d, $\left.{ }^{1} \mathrm{~J}(\mathrm{C}, \mathrm{D}) 10.3 \mathrm{~Hz}\right), 129.5,129.9,133.0,158.6,159.1 \mathrm{ppm}$; IR $\left(\mathrm{CHCl}_{3}\right): \bar{v}=3053,2930,1656,1589,1499,1264$, $1110 \mathrm{~cm}^{-1} ; \mathrm{MS}\left(\mathrm{NH}_{4}^{+}\right): \mathrm{m} / \mathrm{z}: 263\left[\mathrm{M}+\mathrm{NH}_{4}^{+}\right]$; elemental analysis calcd (\%) for $\mathrm{C}_{15} \mathrm{H}_{15} \mathrm{O}_{3} \mathrm{D}$ (245.29): $\mathrm{C} 73.46, \mathrm{H}$ 6.57, O 19.57 found C 73.71, H 6.58, O 19.51. 


\section{Acknowledgements}

We thank the Ministère de la Recherche et de l'Enseignement for financial support of this work through an MRE grant to J.-M.B. and A.G.. We also acknowledge Jean-Pierre Baltaze for NMR spectroscopy; Alain Valleix for mass spectroscopy; and Jean Roger Desmur, Thierry Schlama, and Sylvie Jost from Rhodia Organique Fine for helpful discussions.

\section{References}

[1] a) G. R. Brown, D. S. Clarke, A. J. Foubister, S. Freeman, P. J. Harrison, M. C. Johnson, K. B. Mallion, J. McCormick, F. McTaggart, A. C. Reid, G. J. Smith, M. J. Taylor, J. Med. Chem. 1996, 39, 2971 - 2979; b) Y. Tamura, F. Watanabe, T. Nakatani, K. Yasui, M. Fuji, T. Komurasaki, H. Tsuzuki, R. Maekawa, T. Yoshioka, K. Kawada, K. Sugita, M. Ohtani, J. Med. Chem. 1998, 41, 640 - 649; c) M. L. Quan, A. Y. Liauw, C. D. Ellis, J. R. Pruitt, D. J. Carini, L. L. Bostrom, P. P. Huang, K. Harrison, R. M. Knabb, M. J. Thoolen, P. C. Wong, R. R. Wexler, J. Med. Chem. 1999, 42, 2752 - 2759; d) W. V. Ruyle, H. Jones, A. R. Matzuk, K. W. Kelly, B. E. Witzel, W. J. Holtz, R. A. Houser, T. Y. Shen, L. H. Sarett, J. Med. Chem. 1978, 21, 1093 - 1099; e) M. C. Chrysselis, E. A. Rekka, P. N. Kourounakis, J. Med. Chem. 2000, 43, 609 - 612; f) K. Kawada, A. Arimura, T. Tsuri, M. Fuji, T. Komurasaki, S. Yonezawa, A. Kugimiya, N. Haga, S. Mitsumori, M. Inagaki, T. Nakatani, Y. Tamura, S. Takechi, T. Taishi, J. Kishino, M. Ohtani, Angew. Chem. 1998, 110, 1015 - 1017; Angew. Chem. Int. Ed. 1998, 37, 973 - 975; g) Y. F. Hallock, J. H. Cardellina II, M. Schäffer, G. Bringmann, G. François, M. R. Boyd, Bioorg. Med. Chem. Lett. 1998, 8, 1729 - 1734; h) J. Milton, M. J. Slater, A. J. Bird, D. Spinks, G. Scott, C. E. Price, S. Downing, D. V. S. Green, S. Madar, R. Bethell, D. K. Stammers, Bioorg. Med. Chem. Lett. 1998, 8, 2623 - 2628; i) G. Bringmann, J. Holenz, R. Weirich, M. R, benacker, C. Funke, Tetrahedron 1998, 54, 497 - 512.

[2] J. L. Schulte, S. Laschat, V. Vill, E. Nishikawa, H. Finkelmann, M. Nimtz, Eur. J. Org. Chem. 1998, 2499 - 2506.

[3] A. C. Spivey, T. Fekner, S. E. Spey, J. Org. Chem. 2000, 65, 3154 - 3159.

[4] J. Hassan, M. Se-vignon, C. Gozzi, E. Schulz, M. Lemaire, Chem. Rev. 2002, 102, 1359 - 1469.

[5] P. E. Fanta, Synthesis 1974, 9 - 21.

[6] M. Gomberg, W. E. Bachmann, J. Am. Chem. Soc. 1924, 46, 2339 - 2343.

[7] J. K. Stille, Angew. Chem. 1986, 98, 504 - 519; Angew. Chem. Int. Ed. Engl. 1986, 25, 508 - 524.

[8] a) N. Miyaura, A. Suzuki, Chem. Rev. 1995, 95, 2457 - 2483; b) K. Tamao, K. Sumitani, M. Kumada J. Am. Chem. Soc. 1972, 94, 4374 - 4376; c) K. J. P. Corriu, J. P. Masse, J. Chem. Soc. Chem. Commun. 1972, 144 - 146.

[9] a) R. A. Benkeser, R. G. Severson, J. Am. Chem. Soc. 1949, 71, 3838 - 3839; b) R. A. Benkeser, W. E. Buting, J. Am. Chem. Soc 1952, 74, 3011 - 3014; c) J. D. Roberts, H. E. Simmons, L. A. Carlsmith, C. W. Vaughan, J. Am. Chem. Soc 1953, 71, 3290 - 3291; d) R. A. Benkeser, G. Schroll, J. Am. Chem. Soc. 1953, 75, 3196 - 3197; e) E. R. Biehl, A. Razzuk, M. V. Jovanovic, S. P. Khanapure, J. Org. Chem. 1986, 51, 5157 - 5160; f) A. T. Bottini, J. D. Roberts, J. Am. Chem. Soc. 1957, 79, 1458 - 1462; g) R. W. Hoffmann, Chem. Rev. 1965, 65, 98, 222 - 234; h) G. Wittig, R. W. Hoffmann, Chem. Ber. 1962, 2729 - 2734; i) H. Hart, K. Harada, C.-J. F. Du, J. Org. Chem. 1985, 50, 3104 - 3110; j) A. Saednya, H. Hart, Synthesis 1996, 1455 - 1458; k) R. Huisgen, J. Sauer, Angew. Chem. 1960, 3, 91 - 126.

[10] A. Adejare, D. D. Miller, Tetrahedron. Lett. 1984, 25, 5597 - 5598.

[11] L. Friedman, J. F. Chlebowski, J. Am. Chem. Soc. 1969, 91, 4864 - 4871.

[12] A. I. Meyers, P. D. Pansegrau, Tetrahedron. Lett. 1983, 24, 4935 - 4938.

[13] J. L. Schulte, S. Laschat, Synthesis 1999, 3, 475 - 478.

[14] F. W. Bergstrom, R. E. Wright, C. Chandler, W. A. Gilkey, J. Org. Chem. 1936, 1, 170 - 178.

[15] a) H. Gilman, E. A. Zoellner, W. M. Selby, J. Am. Chem. Soc. 1933, 55, 1252 - 1257; b) T. Oshiki, T. Imamoto, Bull. Chem. Soc. Jpn. 1990, 63, 3719 - 3721.

[16] W. E. Truce, E. Wellisch, J. Am. Chem. Soc. 1952, 74, 5177 - 5179.

[17] a) G. M. Shutske, R. C. Allen, M. F. Försch, L. L. Setescak, J. C. Wilker, J. Med. Chem. 1983, 26, 20, 1307 - 1311;

b) M. P. Doyle, W. J. Bryker, J. Org. Chem. 1979, 44, 1572 - 1574; c) J. B. Hendrickson, J. Am. Chem. Soc. 1961, 83, 1251.

[18] A. I. Meyers, J. R. Flisak, R. A. Aitken, J. Am. Chem. Soc. 1987, 109, 5446 - 5452. 